\section{ANALYTICAL HIERARCHY PROCESS AND MARKOV CHAIN IN SHARED KNOWLEDGE THROUGH SOCIAL MEDIA}

\author{
Nor Intan Saniah Sulaimana, Saadiah Ghazalia*,Nerda Zura \\ Zabidia, Mohd Faizal Omara, Rose Alinda Alias ${ }^{b}$
}

aSchool of Quantitative Sciences, UUM College of Arts and Sciences, UUM, Sintok, Kedah, Malaysia bFaculty of Computing, Universiti Teknologi Malaysia, 81310 UTM Johor Bahru, Johor, Malaysia
Article history

Received

02 June 2015

Received in revised form

9 June 2015

Accepted

1 September 2015

*Corresponding author saadiahsaadiah@gmail.com

\section{Graphical abstract}

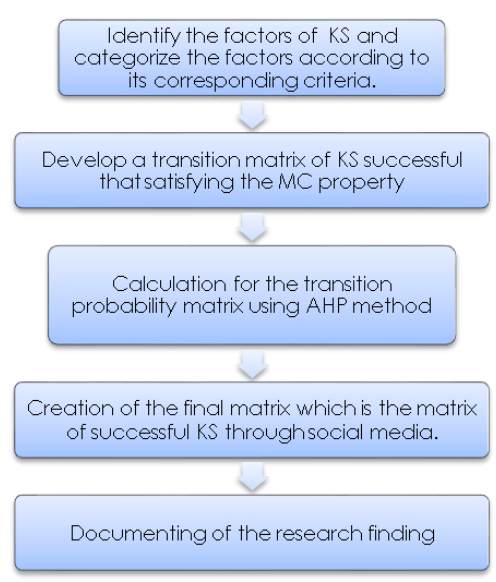

\begin{abstract}
Many studies have been performed to measure successful knowledge sharing in general. However, limited study has been done to assess successful knowledge sharing through social media. Hence, in this paper intend to discuss our approach to assess knowledge sharing among personal social media user. In order to achieve our objective, we proposed to integrate Analytic Hierarchy Process (AHP) and Markov Chain (MC) technique to investigate the pattern of the shared knowledge through social media. Markov Chain will be used to model the knowledge sharing success through expert opinion and stochastic process. We anticipate the outcome of the assessment in a form of a final matrix showing the probability of successful knowledge sharing through social media. The elements in each row of the Markov Chain transition matrix will be calculated using Analytic Hierarchy Process. The assessment tool produce from our research is expected to benefit policy maker or internet user in order to enhance their knowledge sharing strategy in social media application.
\end{abstract}

Keywords: Knowledge sharing; social media; analytic hierarchy process; Markov Chain

(C) 2015 Penerbit UTM Press. All rights reserved

\subsection{INTRODUCTION}

Each individual has their own preference and method to deliver knowledge to their friends, families and communities. In this modern world, knowledge is easily shared through social media. As of March 2012, there were over 835 million registered users of Facebook worldwide (Internet World Stats, 2012), indicating a large number of Facebook users. Despite comprehensive studies that have used discrete theoretical viewpoints to gather knowledge sharing (KS), successful KS is still a dilemma [1]. Thus, this contributes on the lack of number and validity of shared knowledge. Conflicts of interest, knowledge hoarding and lack of psychological understanding are among the potential reasons for the lack of $\mathrm{KS}$ [2][3][4][5].

Many influential factors determine the success of KS. Knowing the success rate of $\mathrm{KS}$ is very important because it serves as an essential element of knowledge management (KM) among coordinated organizations. This paper proposes a prediction framework based on the Analytic Hierarchy Process (AHP) and Markov Chain (MC) theory to forecast the success or failure values of KS. The next two sections briefly discusses about KS and social media. AHP and Markov Chain theory is derived in Section IV which will also briefly explain the methodology use in this review paper. Finally, discussion and conclusion are given in Section $\mathrm{V}$. 


\subsection{KNOWLEDGE SHARING (KS)}

Knowledge sharing can be described as a process of communication whereby two or more parties are involved in the transfer of knowledge [6]. This is a process that involves the provision of knowledge by a source, followed by the interpretation of the communication by one or more recipients. The output of the process is the creation of new knowledge.

Hence, KS is defined as a process of communication between two or more participants involving the provision and acquisition of knowledge. KS process is varying depending on the number of its residing entities. In large multinational organizations, KS requires a complicated process due to the need to negotiate and understand among diverse individuals as well as larger groups and collectives [7].

\subsection{Social Media}

Shared knowledge can be applied with anyone, everywhere and anywhere. The methods include discussion in group, informal face-to-face meetings or using technology applications such as through social media (also referred as Web 2.0). Based on Wikipedia, Web 2.0 is a term describing changing trends in the use of World Wide Web technology and web design that aims to enhance creativity, information sharing, collaboration and functionality of the web.

Social media or social network sites make it possible to connect with other people online, examples like Facebook, Hyves, and Linkedln [8]. Boyd \& Elisson [9] did not specifically see social media sites as active networking places, but more as sites were users display their network. As example, Wikipedia is good social network sites to display the networking in socialization. Wiki's serves as a platform to share knowledge and ideas worldwide.

\subsection{METHODS}

Generally for this review paper, qualitative method includes interview, content analysis and literature review are used to investigate and identify the factors of KS through social media. These factors then categorize into their respective criteria.

In the next stage followed by quantitative method Markov Chain, is used to formulate the matrix of KS successful through social media. A transition matrix of successful KS is formulated by obeying the Markov Chain property. The following figure illustrates our approach to develop an assessment tool for successful knowledge sharing in social media.

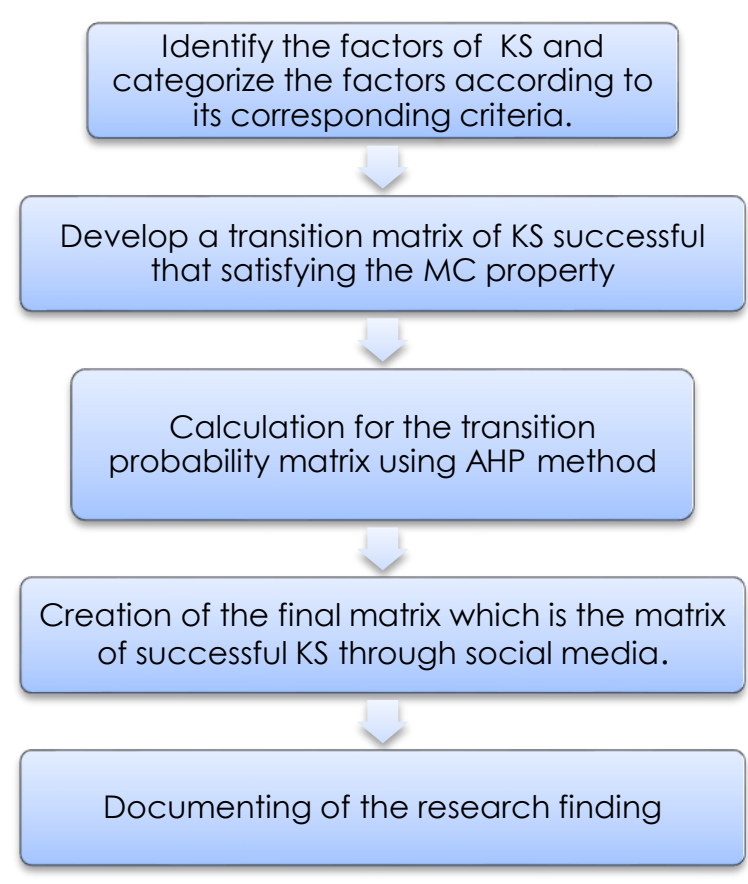

Figure 1 Research review flowchart

Based on the Figure 1 above, the first step on this study is to identify and examine the factors of KS through social media. Literature review analysis will be the main approach in finding the factors. Then, the factors were categorized according to its agreed criteria. A total of five criteria are proposed for this study. To avoid any problem in consistency of the judgments, the number of criteria should be between 5 to 7 [10].

The transition matrix of KS in social media is constructed by understanding and fulfilling the Markov property. The next stage, to calculate the probability for each row of the transition matrix, AHP method is applied. The pairwise comparison of the factors is gathered through series of interview with the academician who is active in using social media such as Facebook to share their knowledge. After completing calculating the transition matrix, the final matrix of successful KS is expected to achieve. Lastly, the process of documenting the finding will be done. Brief definition of Markov chain theory is explained in the next subsection.

\subsection{Markov Chain (MC)}

Theory of Markov Chain (MC) is developed by the Russian mathematician Andreyevich Markov (1856-1922). A MC is defined as a stochastic process that fulfilling the Markov property. The term MC can be refer to the sequence of random variables such a process moves through one state to another. It can be used for describing systems that follow a chain of linked events, where what happens next depends only on the current state of the system. 
The parameter can be discrete state space and a discrete or continuous parameter space. As for this paper, the parameter space represents time and is considered to be discrete.

In constructing a problem or system that exhibits Markov property, a transition matrix based on transition probabilities obtain from that system need to be form first. If a $M C$ has $k$ possible states label as $1,2, \ldots, k$, then the probability of the system is in state-i at any observation after it was in state $-j$ at the preceding observation is denoted by

$$
P_{i, j}=\mathrm{P}\left(X_{n+1}=j \mid X_{n}=i\right)
$$

and is called the transition probability from state $j$ to state i.

They are denoted as the transition matrix $\mathrm{P}$. For $k$ states $\mathrm{P}$ has the following form:

$$
P=\left(\begin{array}{lllc}
p_{11} & p_{12} & \ldots & p_{1 k} \\
p_{21} & p_{22} & \cdots & p_{2 k} \\
& \vdots & \ddots & \vdots \\
p_{k 1} & p_{k 2} & \cdots & p_{k k}
\end{array}\right)
$$

Clearly, the quantities $P_{i j}$ satisfy the conditions

$$
\begin{array}{ll}
P_{i j} \geq 0 & \text { for } i, j=0,1,2 \ldots \ldots \\
\left.\sum_{j=0}^{\infty} P_{i j}=1 \quad \text { for } i, j=0,1,2, \ldots . . \text { (Eq. } 3\right)
\end{array}
$$

Predictions of future state probabilities can be calculated by solving the matrix equation:

$$
P^{(n)}=P^{(n-1)} \cdot P
$$

With increasing time steps, a MC may approach a constant state probability vector, which is called limiting distribution:

$\lim _{n \rightarrow \infty} \operatorname{Pr}\left\{X_{n}=k \mid X_{0}=j\right\}=\pi_{k}>0, \quad$ for $k=0,1, \ldots$

The AHP method is used to calculate probability distribution in each row of the transition matrix. Brief explanation on AHP is explained in the next subsection.

\subsection{Analytic Hierarchy Process (AHP)}

We have to calculate the probability distribution in each row of matrix $P$ that has to satisfy the Markov property. The method that is used in this paper is AHP. AHP is a structured technique for organizing and analyzing complex decisions. This method was developed by Thomas L. Saaty in 1970s. AHP helps decision makers in finding one that best suits their goal and their understanding of the problem.

Other than that, it provides a comprehensive and rational framework for structuring a decision problem and for evaluating alternative solutions. Its main characteristic is that it is based on pair wise comparison [11]. The primary advantage of the AHP is ability to use pair wise comparisons to obtain a ration scale of measurement which makes comparison among alternatives and measurement of both tangible intangible factors [12].

To calculate the probability for the matrix at each level, the pair wise comparisons are organized into a matrix and the weights of the items being compared are determined by computing the maximum eigenvalue of the matrix. A weighted averaging approach is used to combine the results across levels of the hierarchy to compute the final weight for each alternative.

Table 1 AHP table for weighting factors

\begin{tabular}{|l|l|}
\hline & \multicolumn{1}{|c|}{$\mathrm{i}^{\text {th }}$ factor } \\
\hline $\mathrm{j}^{\text {th }}$ factor & $\begin{array}{l}\text { Wije comparing } \mathrm{i}^{\text {th }} \text { factor to } \mathrm{j}^{\text {th }} \text { factor (the } \\
\text { ratio of importance of } \mathrm{i}^{\text {th }} \text { factor to } \mathrm{j}^{\text {th }} \\
\text { factor) }\end{array}$ \\
\hline
\end{tabular}

A pair wise comparison for factors of knowledge sharing is obtained through series of interviews. The interviewee is the academician that is active in using Facebook page as their medium of knowledge sharing.

At the end of this review paper, a decision hierarchy with the goal of achieving KS successful is expected to be achieved. The subordinate of the goal is the criteria factors of KS. The last line of the hierarchy is the state of KS (Figure 2).

Apart from that, a collection of academician post's which have been analyzed qualitatively based on intuitive model is used to obtain the primary information of knowledge sharing factors. Intuitive model is chosen because this model provides a quick evaluation while it helps to ensure in examining the factors that are appropriate for this review paper.

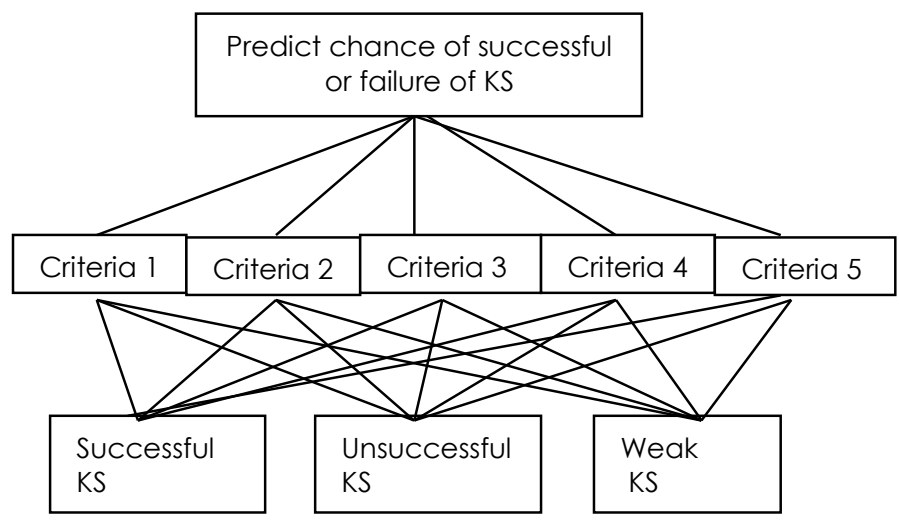

Figure 2 Decision hierarchy of successful knowledge shared 
From the figure above, we develop the transition matrix of KS. Three different states are considered for knowledge sharing Set of $P$ has three members \{successful sharing, unsuccessful sharing and weak sharing\}. Thus matrix $P$ is a $3 \times 3$ matrix (Figure 3).

In this review paper, the propose transition matrix is as follow:

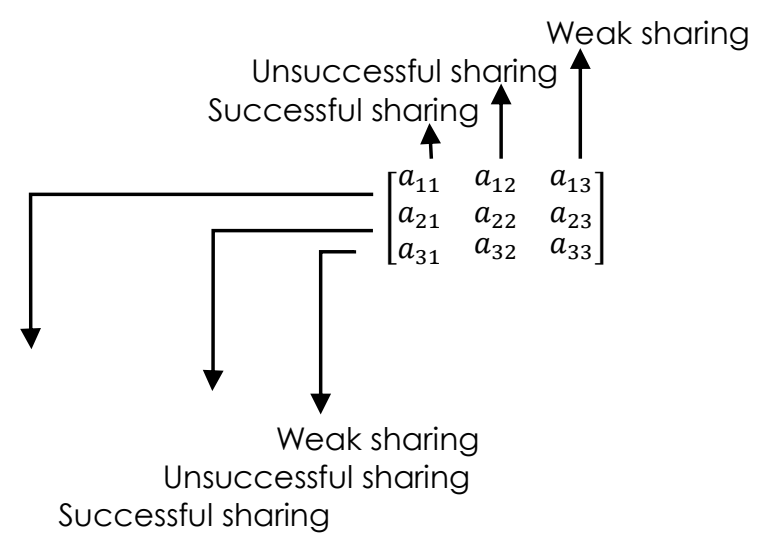

The final matrix that is expected to be developed at the end of this research in the form as shown below:

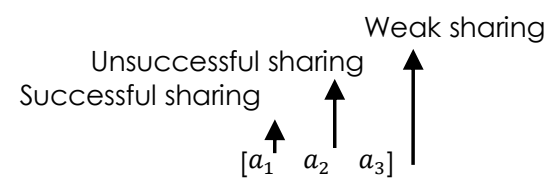

Figure 3. Propose transition matrix of successful shared knowledge

\subsection{DISCUSSION}

Research by [12] found that learning, training, culture and structure of organization are among the important criteria needed in predicting successful KS. Their paper also discussed the findings on the transition matrix resulted from the AHP calculation. Figure below show the value for each element in the transition matrix.

$\left[\begin{array}{cccc}0.357 & 0.25 & 0.214 & 0.179 \\ 0.625 & 0.188 & 0.125 & 0.062 \\ 0.435 & 0.044 & 0.304 & 0.217 \\ 0.258 & 0.328 & 0.220 & 0.194\end{array}\right]$

Figure 4 Value For Each Element In Transition Matrix (adopted From [12])

Our research is different from [12] as we are aiming to model the successful of KS specifically through social media. We are considering new factors that contribute the implementation of knowledge sharing by means of social media. Moreover, most of pass studies concentrate on knowledge management (KM) is successful case.
For example, ref [13] described that there are seven criteria applied in measuring the successful implementation of KM. These criteria are employee traits, strategy, superintendent traits, audit and assessment, organizational culture, operation procedure and information technology. They used Fuzzy Multi Criteria Decision Making (FMCDM) to predict the successful KM. Throughout their research, the result indicates that the possibility of successful knowledge management implementation (0.70127) is generally twice that of unsuccessful knowledge management implementation (0.29873). Thinking about the likelihood of KM task achievement or failure is deficient if the likelihood of $K S$ is obscure. Therefore, we try to demonstrate a new model of KS using a different approach from the previous studies.

\subsection{THE WAY FORWARD}

The future direction from this review paper is to model the successful of KS through social media in a matrix form. Most of pass studies focusing on knowledge management (KM) successful. Knowing about the possibility of KM project success or failure is inadequate if the possibility of KS is unknown. Therefore, we try to demonstrate a new model of KS using a different approach from the previous studies. Apart from that, this review paper is different from the previous studies as it considers the effect of social media on KS.

\subsection{CONCLUSION}

As a conclusion, this review paper can help the decision maker to make much more effective decision in order to increase or decrease the investment in KS. Besides that, it can provides decision makers with useful information to make decision regarding whether to initiate $\mathrm{KM}$, inhibit adoption or undertake some remedial improvement actions to increase the possibility of successful KM implementation.

\section{Acknowledgement}

This study was supported by the Research Acculturation Collaborative Effort (RACE) Funds, Ministry of Education (No. 600-RMI/RACE 16/6/2(2/2013.

\section{References}

[1] Wang, S.,Noe, R. A., 2010. Knowledge Sharing: A Review and Directions for Future Research, Human Resource Management Review. 20(2) : 115-131

[2] Becerra-Fernandez ,I., Sabheral. R., 2010. Knowledge Management: Systems and Processes,New York: ME Sharpe, 5660 
[3] Cabrera, A., Collins, W. C., Salgado, J. F., 2006. Determinants of Individual Engagement InKnowledge Sharing, The International Journal of Human Resource Management. 17( 2): 245-264

[4] Cho, N., Li G., Su, C. J., 2007. An Empirical Study on The Effect of Individual Factors on Knowledge Sharing by Knowledge Type, Journal of Global Business and Technology. 3( 2): 1-15

[5] Matzler, K., Mueller, J., 201 1.Antecedents Of Knowledge Sharing Examining The Influence Of Learning And Performance Orientation, Journal of Economic Psychology, 32 (3): 317-329

[6] Abel Usoro, M. , Sharratt, W., Tsui, E., Shekhar, S., 2007. Trust as an Antecedent to Knowledge Sharing In Virtual Communities of Practice, Knowledge Management Research \& Practice. 5: 199212

[7] Ellison, N. B. , Gibbs, J. L., Weber, M. S., 2014 . The Use of Enterprise Social Network Sites for Knowledge Sharing in Distributed Organizations: The Role Of Organizational Affordances, American Behavioral Scientist. 1-21
[8] Koeleman, H., 2009. The Managers are not Leaders.[Online]. Retrieved from http://www.adformatie.nl

[9] Boyd, D. M., Ellison, N. B., 2007. Social Network Sites: Definition, History, and Scholarship, Journal of Computer-Mediated Communication. 13: 210-230

[10] Klaus, 2013 .Business Performance Management.[Online]. Retrieved from http://bpmsg.com/ahp-online-calculator/

[11] Ngai, E.W.T., Chan, E.W.C., 2005 . Evaluation of Knowledge Management Tools using AHP, Expert Systems with Applications. 29(4): 889-899

[12] Aminifard N., Afrazeh, A., 2008. A Method of Presentation of Knowledge Sharing, based on the Markov Chain Theory, World Applied Sciences Journal. 3(2): 50-54

[13] T. H. Chang, and, T. C. Wang, 2009.Using The Fuzzy Multi-Criteria Decision Making Approach For Measuring The Possibility Of Successful Knowledge Management, Information Sciences. 179(4): 355-37 